\title{
Heterogeneous distribution of Plasmodium falciparum drug resistance haplotypes in subsets of the host population Sonja Schoepflin ${ }^{1}$, Jutta Marfurt ${ }^{1}$, Mary Goroti ${ }^{2}$, Moses Baisor $^{2}$, Ivo Mueller ${ }^{2}$ and Ingrid Felger*1
}

\author{
Address: ${ }^{1}$ Swiss Tropical Institute, Department of Medical Parasitology and Infection Biology, Socinstr.57, 4002 Basel, Switzerland and 2Papua \\ New Guinea Institute of Medical Research, Madang, P.O. Box 378, M.P. 511, Papua New Guinea \\ Email: Sonja Schoepflin - s_schoepflin@hotmail.com; Jutta Marfurt - jutta.marfurt@gmail.com; Mary Goroti - mary.goroti@pngimr.org.pg; \\ Moses Baisor - yagaum@pngimr.org.pg; Ivo Mueller - ivomueller@fastmail.fm; Ingrid Felger* - ingrid.felger@unibas.ch \\ * Corresponding author
}

Published: 6 May 2008

Malaria Journal 2008, 7:78 doi:10.1 186/1475-2875-7-78

This article is available from: http://www.malariajournal.com/content/7///78

(C) 2008 Schöpflin et al; licensee BioMed Central Ltd.

This is an Open Access article distributed under the terms of the Creative Commons Attribution License (http://creativecommons.org/licenses/by/2.0), which permits unrestricted use, distribution, and reproduction in any medium, provided the original work is properly cited.
Received: 18 January 2008

Accepted: 6 May 2008

\begin{abstract}
Background: The emergence of drug resistance is a major problem in malaria control. For mathematical modelling of the transmission and spread of drug resistance the determinant parameters need to be identified and measured. The underlying hypothesis is that mutations associated with drug resistance incur fitness costs to the parasite in absence of drug pressure. The distribution of drug resistance haplotypes in different subsets of the host population was investigated. In particular newly acquired haplotypes after radical cure were characterized and compared to haplotypes from persistent infections.
\end{abstract}

Methods: Mutations associated with antimalarial drug resistance were analysed in parasites from children, adults, and new infections occurring after treatment. Twenty-five known single nucleotide polymorphisms from four Plasmodium falciparum genes associated with drug resistance were genotyped by DNA chip technology.

Results: Haplotypes were found to differ between subsets of the host population. A seven-fold mutated haplotype was significantly reduced in adults compared to children and new infections, whereas parasites harbouring fewer mutations were more frequent in adults.

Conclusion: The reduced frequency of highly mutated parasites in chronic infections in adults is likely a result of fitness costs of drug resistance that increases with number of mutations and is responsible for reduced survival of mutant parasites.

\section{Background}

The emergence of drug resistance poses a major problem for malaria control. Research has focused mainly on elucidating the mode of action of antimalarial drugs and on the molecular mechanisms leading to drug resistance. The development of drug resistance mostly involves single nucleotide polymorphisms (SNPs) in genes encoding the drug targets, such as metabolic enzymes or transmembrane transporters. Molecular studies have identified a number of SNPs in the Plasmodium falciparum multi-drug resistance gene 1 ( $p f m d r 1$ ), the chloroquine resistance transporter ( $p f c r t)$, the dihydrofolate reductase ( $p f d h f r)$ and the dihydropteroate synthase ( $p f d h p s$ ) that were associated with drug resistance against the most commonly 
used antimalarials chloroquine (CQ) and the combination of antifolate sulphadoxine/pyrimethamine (SP). Allelic exchange experiments did provide direct evidence for the role of pfdhfr in resistance to pyrimethamine [1], pfdhps in resistance to sulphadoxine [2] and the role of pfcrt in resistance to CQ [3]. Reed et al [4] could show that mutations in pfmdr1 are not essential, but can modulate the level of drug resistance against CQ. Evidence from molecular epidemiological field studies confirmed associations of the mutations K76T [5-7] and N86Y [8-10] with in vivo drug resistance against $C Q$, whereas different combinations of mutations in $d h f r$ and $d h p s$ were associated with in vivo drug resistance against SP [for example [1114]].

A number of mathematical models have been designed to predict the transmission and spread of drug resistance [15-20]. Questions addressed include the role of transmission intensity on the spread of drug resistance or the possibility of a preferential transmission of resistant versus sensitive parasites. The effect of transmission intensity on the spread of drug resistance was discussed controversially $[19,21]$. A more recent review by Hastings \& Watkins [15] proposed that transmission intensity does not have a direct impact on the evolution of drug resistance, but directly determines the dynamics of resistance via "effectors", such as intra-host competition, level of drug use in the population, extent of sexual recombination, proportion of malaria infections treated, or number of parasites in a human host.

Different authors studied the preferential transmission of resistant versus sensitive parasites. It has been proposed that moderately mutated parasites that result in high levels of parasitological failures following SP treatment, have a greater transmission potential than highly mutated parasites, because the latter are more likely to cause clinical failure within a short time after treatment and are therefore more often subject to rescue treatment [22]. A number of field studies have reported increased gametocyte carriage in blood of CQ or SP treated individuals [2327] or in individuals carrying drug resistant strains $[23,25]$. However, these findings have been questioned by lacking evidence that parasites post SP-treatment fully develop to infective stages in the mosquito [28].

The field study presented here was conducted in Papua New Guinea (PNG) and investigated the distribution of drug resistance genotypes in subsets of the host population. In particular, the actually transmitted genotypes were characterized. This was possible by following individuals after radical cure treatment. The first $P$. falciparum positive blood samples of these individuals were genotyped for all known markers for drug resistance.
The underlying hypothesis of this study was that new infections following radical cure are not yet subject to major selective constraints, because these newly arriving infections do not have to compete against already persisting infections. Here all transmitted genotypes irrespective of their fitness are expected to be found. In contrast, parasites in asymptomatic chronic infections from community samples have been subject to within-host competition and differential fitness is likely to affect their long-term survival in the host. Asymptomatic individuals are therefore expected to carry a lower prevalence of mutated genotypes as a result of decreasing fitness with increasing number of mutations.

By molecular epidemiological studies in Madang, PNG, detailed information has been provided on the currently high levels of mutation rates in Pfcrt K76T (97\%), Pfmdr1 N86Y (96\%) and Pfdhfr S108N (82\%) and C59R (74\%)[29]. Another study in PNG observed a significant increase in the $d h f r$ double mutant C59R + S108N over a period of two years $(83 \%$ to $96 \%)$. This study was performed in 2002 and 2003, a few years after the introduction of SP as first line treatment in combination with 4aminoquinolines [30]. They also found an increase in the quadruple mutant $d h f r \mathrm{C} 59 \mathrm{R}+\mathrm{S} 108 \mathrm{~N}+$ dhps A437G + K540E from $0 \%$ to $8.2 \%$ and even though this was not significant, these results suggest that resistance to SP is rapidly developing in PNG. The genotype most frequently found in treatment failures contained a quadruple mutant in pfcrt $(\mathrm{K} 76 \mathrm{~T}+\mathrm{N} 326 \mathrm{D}+\mathrm{I} 356 \mathrm{~L}+\mathrm{A} 220 \mathrm{~S})$ in combination with the pfmdr1 mutant N86Y and the double pfdhfr mutant S108N+C59R.

For the present investigation, which aims primarily at multi-loci haplotypes, the PNG field site provides optimal conditions. A haplotype, which is here defined as the genetic make up of an individual parasite clone at 25 loci from four marker genes of drug resistance, can be directly deduced after genotyping a single clone infection. As multiclonal infections are rare in PNG, with a mean multiplicity of infection (MOI) between 1.3 and 1.8 [31-33], the high prevalence of single-clone infections greatly facilitated the study of drug resistance haplotypes.

\section{Materials and methods}

The present study made use of two sets of blood samples: (i) cross-sectional surveys including Papua New Guinean individuals of all ages, and (ii) first $P$. falciparum positive samples from a follow up after radical cure with artesunate in five to 14 year-old children. Both sets of samples were collected in the same villages in PNG, in the years $2003-2005$.

In the treatment to reinfection study (TRS) in 2004/2005 [for details see [34]] a total of 206 children from five to 14 
years of age were enrolled at the Mugil and Megiar elementary schools which are situated about $50 \mathrm{~km}$ north of Madang town, PNG. After obtaining written informed consent from the parents or guardians each child was clinically examined, two blood slides (thick and thin films) were prepared for microscopical determination of malaria infections and a venous blood sample was collected at baseline. Subsequently, all children were treated with a seven-day course of artesunate monotherapy according to PNG national treatment guidelines (i.e. $4 \mathrm{mg} / \mathrm{kg}$ at day 1 , $2 \mathrm{mg}$ at days 2-7). After treatment, two-weekly active follow ups were conducted at the schools to check for new malaria infections and presence of febrile illness. Therefore, each child was clinically assessed, a rapid diagnostic test (RDT) (ICT Diagnostics, South Africa) was performed, blood slides were prepared and $250 \mu \mathrm{l}$ of blood were collected by finger prick every two weeks.

Positive samples were identified by microscopy and LDRFMA [35] as described in more detail in Michon et al [34]. All baseline and first PCR positive samples after treatment were genotyped for the highly polymorphic marker gene merozoite surface protein 2 ( $m s p 2$ ) and compared by PCR-RFLP [36,37] in order to distinguish new from recrudescent infections. Samples that were typed as recrudescent infection, but were collected as late as 10 weeks or more after the baseline survey, were additionally genotyped for a second marker gene (msp1) [38], because recrudescence seemed to be unlikely after an interval of this length.

In the same villages two household-based cross sectional surveys were conducted in 2003 and 2004 which included participants of all age groups. Upon receiving informed consent a questionnaire was completed for each participant, blood slides prepared for microscopical examination and a venous blood samples was collected for further laboratory analysis. All samples were msp2-genotyped using PCR-RFLP as described above to determine the multiplicity of infection (MOI).

All samples that were determined to be single or double infections by msp2 genotyping were further analysed for mutations in drug resistance genes by DNA chip technology [39]. This method allows parallel identification of 25 single nucleotide polymorphisms (SNPs) that were found to be associated with drug resistance against a number of different antimalarial drugs. It is based on PCR amplification of target sequences within the genes Pfmdr1, Pfcrt, Pfdhfr and Pfdhps. A primer extension reaction with fluorescent labelled ddNTPs follows this PCR step. The extended primers are subsequently hybridized on a microarray carrying the antisense DNA of the extension primers and scanned at different wavelength using an Axon 4100A fluorescent scanner to determine the incorporated
ddNTP. Pictures were acquired and analysed using the Axon GenePix ${ }^{\circledast}$ Pro (version 6.0) software. The codons investigated with this method include N86Y, Y184F, S1034C, N1042D and D1246Y in Pfmdr1, the codons K76T, H97Q, T152A, S163R, A220S, Q271E, N326D/S, I356L/T and R371I in Pfcrt, the codons A16V, N51I, C59R, S108N/T and I164L in Pfdhfr and the codons S436A, A437G, K540E, A581G, I640F and H645P in Pfdhps.

The dataset for statistical analysis consisted of all crosssectional samples that had been genotyped as single-clone infections and all new infections (irrespective of malaria symptoms) plus baseline samples with MOI = 1 from TRS study. Samples with MOI $=2$ were taken into consideration, if it was possible to unequivocally determine the haplotype of these samples, i.e. samples showing a mixed infection for more than two loci were not included. Samples from the cross sectional surveys were grouped into adults ( $>14$ years of age) and children five -14 years of age. All individuals from the household surveys who had received any antimalarial treatment during the last two months prior to the survey were excluded.

For simplified presentation of haplotypes, only SNPs of which the mutated alleles were actually detected in this study area are itemized in the haplotype descriptions, whereas SNPs found only in the wild type form are not listed. Thus, the presented haplotype provides molecular typing information on alleles at the following 11 positions: dhfr59, dhfr 108, dhps437, dhps540, mdr86, mdr184, mdr1042, crt76, crt220, crt326 and crt356. Only samples with a complete set of these 11 polymorphic SNPs were taken into consideration.

To compare the haplotype frequencies between datasets logistic regression statistics was applied.

\section{Results}

Blood samples from radically cured individuals were genotyped for $m s p 2$ in order to distinguish new infections from recrudescent ones and to determine multiplicity of infection (MOI). All new infections with MOI $=1$ or 2 were analysed by DNA Chip to identify SNPs in genes associated with drug resistance. In addition, genotyping on Chip was performed for all single and double infections found in baseline samples of these individuals prior to treatment. Complete haplotype data were obtained for 144 new infections and 109 baseline samples.

From cross sectional surveys, 61 samples with a complete haplotype were grouped as adults $>14$ years and 63 samples derived from children aged five-14 years. Individuals that were treated with antimalarial drugs in the two months prior to the survey were excluded. 
Genotyping was performed using DNA Chip technology [39]. Among the 25 analysed SNPs in the four different genes $P f m d r$, Pfcrt, Pfdhfr and Pfdhps some occurred only as the wild type allele, whereas some mutant alleles had already reached fixation at this field site. For 8 SNPs both the wild type and mutant allele was detected in the samples set.

At this study site a total of 13 different haplotypes were found, all listed in table 1 . The most frequent haplotype in all subsets of the host population was the 7-fold mutated haplotype 'crt76T-crt356L-crt326D-crt220S$d h f r 59 \mathrm{R}-d h f r 108 \mathrm{~N}-m d r 86 \mathrm{Y}$ '. The wild type allele was fixed at the remaining 4 polymorphic codons.

In the samples set, a number of haplotypes occurred at very low frequency (one or two observations). The detection of rare haplotypes depends on sample size and presence or absence of these haplotypes in a population comparison is likely due to chance.

\section{Effect of host age on haplotype frequency}

Haplotype frequencies in children and adults of the cross sectional surveys were compared in order to test for agespecific effects. The 7-fold mutated haplotype 'crt76Tcrt356L-crt326D-crt220S-dhfr59R-dhfr108N-mdr86Y' was the most frequent haplotype in adults and children with a prevalence of $65.57 \%$ and $84.13 \%$, respectively (Table 1 ). The frequency of this haplotype was significantly lower in adults compared to children by more than $20 \%$ (OR =
2.78, $\mathrm{p}=0.02$ ) (Figure 1). In addition to the seven-fold mutated haplotype, seven additional haplotypes were found in adults, most of them occurring at very low frequency with the exception of the 6-fold mutant 'crt76Tcrt356L-crt326D-dhfr59R-dhfr108N-mdr86Y' which showed an increased frequency in adults compared to children (13.11\% vs. $6.35 \%)$. This difference was not statistically significant due to the very few observations.

\section{Haplotypes in new infections}

Since new infections best reflect the haplotype frequencies that are actually transmitted, first infections after radical cure with artesunate monotherapy were genotyped. By genotyping the highly polymorphic $m s p 1$ and $m s p 2$ locus all recrudescent parasites due to treatment failure were excluded. Baseline samples prior to radical cure which had a single or double-clone infection were also genotyped. Figure 2 shows that haplotype frequencies agreed well in baseline samples and new infections, with the exception of some rare types which are probably fluctuating randomly. In addition, haplotype frequencies from age-matched cross sectional samples are indicated and also show good agreement with frequencies found in new infections. The fact that haplotypes in new infections appearing after radical cure, which likely reflect the transmitted parasite population, do not differ from haplotype frequencies in age matched children, suggests a high rate of clone acquisition in children. The concordance between baseline samples and samples from age-matched children proves that the data sets from the cross sectional

Table I: Frequency of haplotypes in different subsets of the host population

\begin{tabular}{|c|c|c|c|c|c|c|c|c|c|c|c|c|c|c|}
\hline \multirow{3}{*}{ Haplotype ${ }^{1}$} & \multirow{2}{*}{\multicolumn{2}{|c|}{$\begin{array}{l}\text { Adults cross } \\
\text { section } \\
(n=61)\end{array}$}} & \multirow{2}{*}{\multicolumn{2}{|c|}{$\begin{array}{l}\text { Children } \\
\text { cross section } 2 \\
(n=63)\end{array}$}} & \multirow{2}{*}{\multicolumn{2}{|c|}{$\begin{array}{c}\text { TRS }{ }^{3} \text { baseline } \\
\text { samples } \\
(n=109)\end{array}$}} & \multirow{2}{*}{\multicolumn{2}{|c|}{$\begin{array}{c}\text { TRS }^{3} \text { new } \\
\text { infections } \\
(n=I 44)\end{array}$}} & \multicolumn{3}{|c|}{ adults vs. children } & \multicolumn{3}{|c|}{ adults vs. new infections } \\
\hline & & & & & & & & & OR & $p^{4}$ & $95 \% \mathrm{Cl}$ & OR & $p^{4}$ & $95 \% \mathrm{Cl}$ \\
\hline & $\mathrm{n}$ & $\%$ & $\mathrm{n}$ & $\%$ & $\mathrm{n}$ & $\%$ & $\mathrm{n}$ & $\%$ & & & & & & \\
\hline crt76 crt326 crt356 & 0 & 0 & 0 & 0 & 0 & 0 & I & 0.69 & - & - & - & - & - & - \\
\hline crt76 crt326 crt356 crt220 & 1 & 1.64 & 1 & 1.59 & 0 & 0 & 0 & 0 & 0.97 & 0.98 & $0.06-15.83$ & - & - & - \\
\hline crt76 crt326 crt356 dhfr 108 dhfr59 & 0 & 0 & 0 & 0 & 3 & 2.75 & 0 & 0 & - & - & - & - & - & - \\
\hline crt76 crt326 crt356 crt220 dhps 540 & 0 & 0 & 1 & 1.59 & & & 0 & 0 & - & - & - & - & - & - \\
\hline crt76 crt326 crt356 crt220 mdr86 & 4 & 6.56 & 2 & 3.17 & 0 & 0 & I & 0.69 & 0.47 & 0.39 & $0.08-2.65$ & 0.1 & 0.04 & $0.01-0.91$ \\
\hline crt76 crt326 crt356 crt220 dhfr 108 dhfr59 & 3 & 4.92 & 0 & 0 & 4 & 3.67 & I & 0.69 & - & - & - & 0.14 & 0.09 & $0.01-1.33$ \\
\hline crt76 crt326 crt356 dhfrl08 dhfr59 mdr86 & 8 & 13.11 & 4 & 6.35 & 15 & 13.76 & 8 & 5.56 & 0.45 & 0.21 & $0.13-1.58$ & 0.39 & 0.07 & $0.14-1.09$ \\
\hline crt76 crt326 crt356 crt220 dhfr 108 mdr86 & 1 & 1.64 & 2 & 3.17 & 2 & 1.83 & 2 & 1.39 & 1.97 & 0.59 & $0.17-22.27$ & 0.85 & 0.89 & $0.75-9.5$ \\
\hline $\begin{array}{l}\text { crt76 crt326 crt356 dhfr } 108 \text { dhfr59 mdr86 } \\
\text { dhps540 }\end{array}$ & 0 & 0 & 0 & 0 & 0 & 0 & I & 0.69 & - & - & - & - & - & - \\
\hline $\begin{array}{l}\text { crt76 crt326 crt356 crt220 dhfrl } 08 \\
\text { dhfr59 mdr86 }\end{array}$ & 40 & 65.57 & 53 & 84.13 & 85 & 77.98 & 126 & 87.5 & 2.78 & 0.02 & $1.18-6.56$ & 3.68 & 0.001 & $1.78-7.57$ \\
\hline $\begin{array}{l}\text { crt76 crt326 crt356 crt220 dhfr } 108 \text { dhfr59 } \\
\text { mdr86 dhps } 540\end{array}$ & 2 & 3.28 & 0 & 0 & 0 & 0 & 4 & 2.78 & - & - & - & 0.84 & 0.85 & $0.15-4.73$ \\
\hline $\begin{array}{l}\text { crt76 crt326 crt356 crt220 dhfr } 108 \text { dhfr59 } \\
\text { mdr } 1042 \text { mdrl } 84\end{array}$ & 1 & 1.64 & 0 & 0 & 0 & 0 & 0 & 0 & - & - & - & - & - & - \\
\hline $\begin{array}{l}\text { crt76 crt326 crt356 crt220 dhfrl } 08 \text { dhfr59 } \\
\text { mdr86 dhps } 437\end{array}$ & I & 1.64 & 0 & 0 & 0 & 0 & 0 & 0 & - & - & - & - & - & - \\
\hline
\end{tabular}

1) Haplotypes represent II single nucleotide polymorphisms (SNPs) in 4 different genes. The remaining 14 SNPs tested but not listed equal to wildtype.

2) Cross-sectional samples include only individuals that have not been treated with antimalarial drugs in the 2 months prior to the survey.

3) TRS, treatment to reinfection study

4) Bold indicates $p<0.05$ 


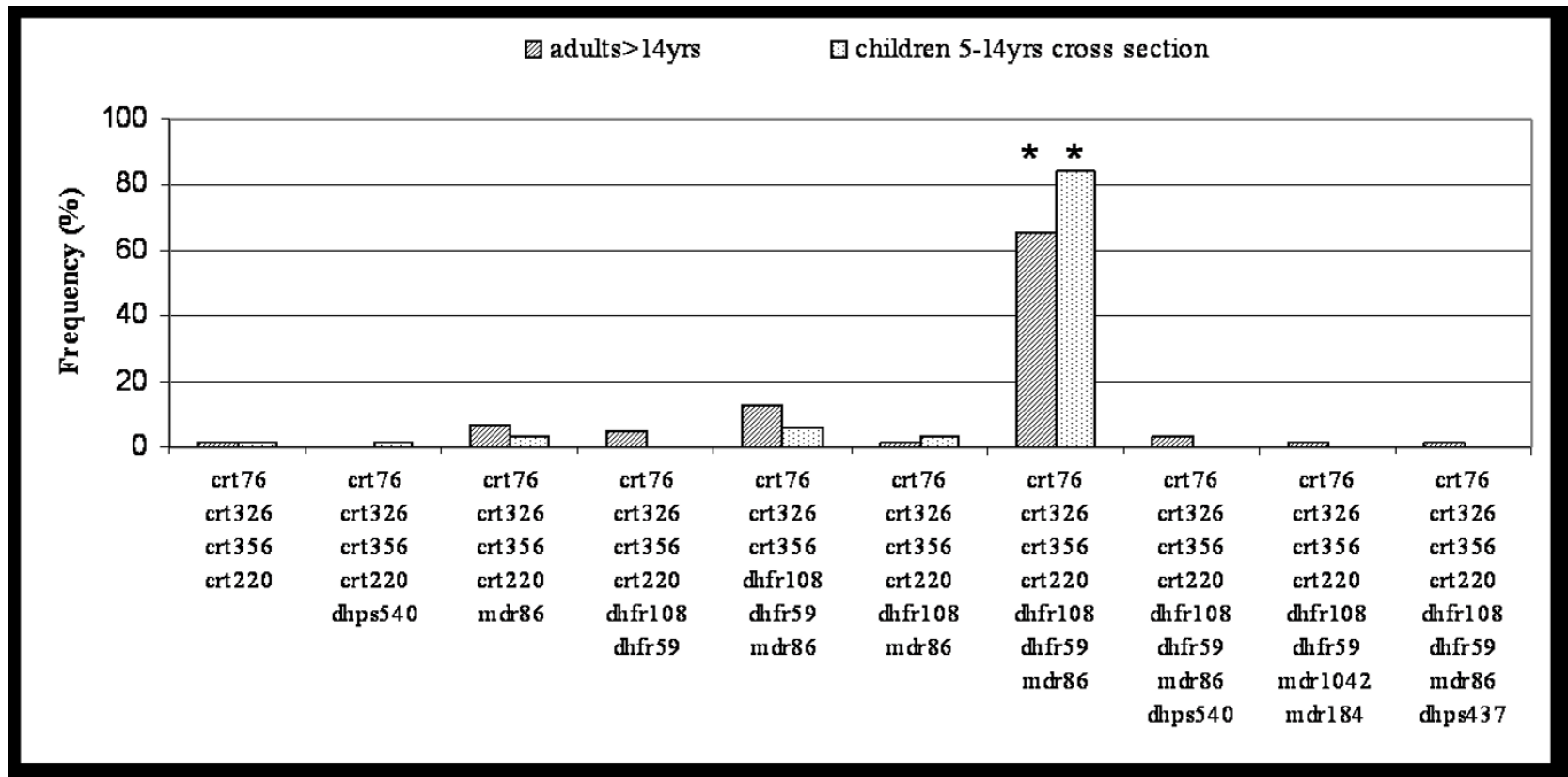

Figure I

Comparison of haplotypes between adults and children of the cross-sectional surveys. * indicates significant difference of haplotype frequency between the two compared groups.

survey and from the radical cure are comparable and unbiased. In all three subsets of the host population from Figure 2, the frequency of the dominant haplotype agreed well and showed no statistically significant difference.

\section{New infections versus persisting infections in adults}

In order to determine whether all haplotypes persist in the host equally well, the haplotype distribution in newly arriving infections in relation to persistent parasites from long term infections were compared. Previously, it was

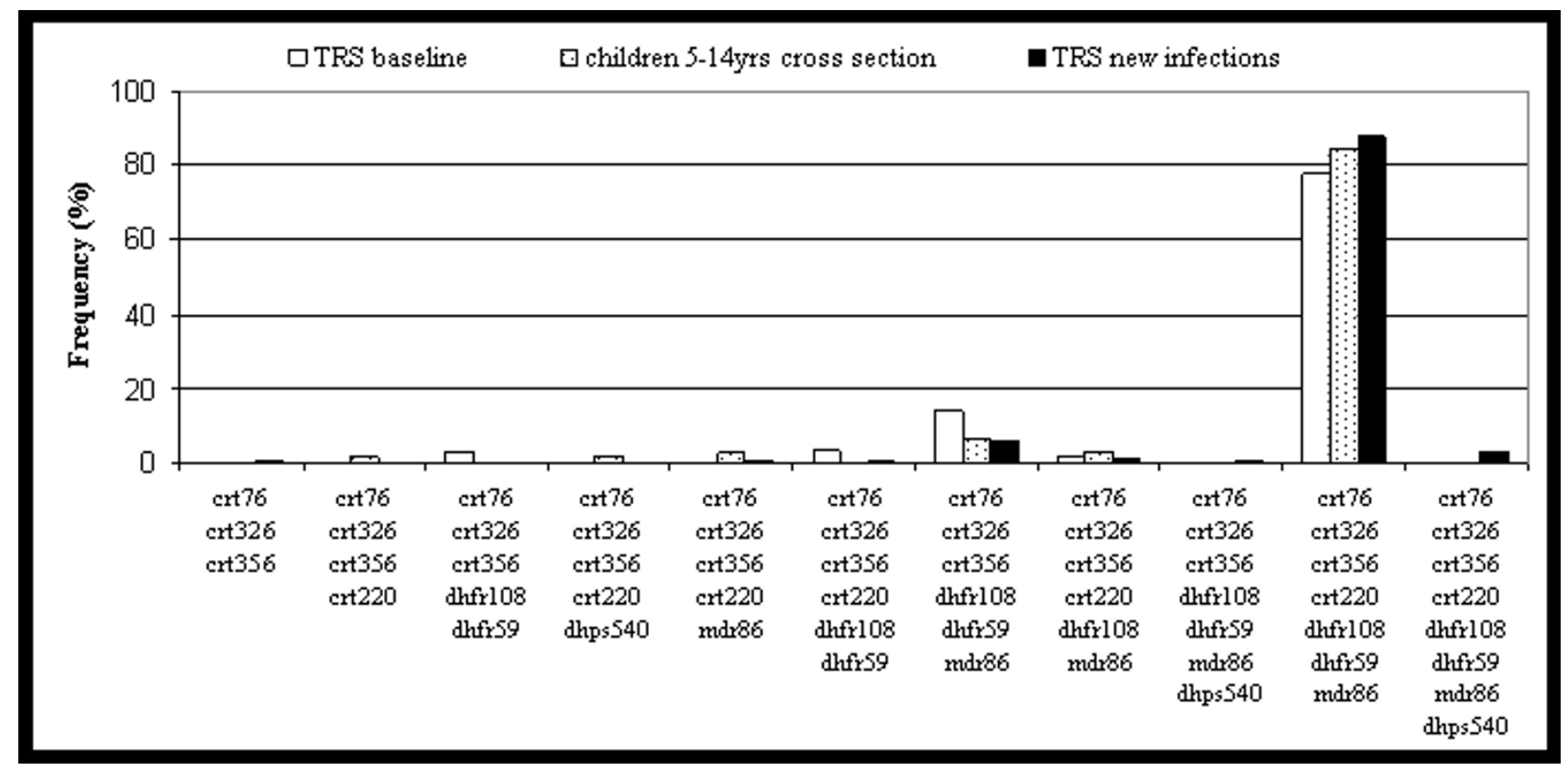

Figure 2

Comparison of haplotypes between children of the cross sectional surveys and baseline samples and new infections from the treatment to reinfection study (TRS). There are no significant differences in haplotype frequencies between groups. 
shown by genotyping P. falciparum clones from a longitudinal study that individual parasite clones can persist over very long time in a semi immune host at very low densities fluctuating around the detection level [40,41]. To select long term persistent infections from this data set, infections from adults $>14$ years were chosen (mean age $=31$ years; $\mathrm{SD}=13.67)$. To avoid any influence of previous drug intake on the prevalence of certain haplotypes, all patients treated with antimalarials two months prior to sampling were omitted from the analysis.

Figure 3 compares new infections and persisting infections in adults. A significantly higher number of sevenfold mutated haplotype was found in new infections compared to frequencies in adults with a difference between the groups of $21.92 \%(\mathrm{OR}=3.68, \mathrm{p}<0.001$; Table 1$)$. In contrast, the frequency of the five-fold mutated haplotype 'crt76T-crt356L-crt326D-crt220S- $m d r 86 \mathrm{Y}^{\prime}$ was significantly lower in new infections $(\mathrm{OR}=0.1, \mathrm{p}=0.04$; Table $1)$. The frequency of 6-fold mutant 'crt76T-crt356Lcrt326D-dhfr59R-dhfr 108N-mdr86Y' was also reduced in new infections, although this difference did not reach statistical significance. This suggests for adults a higher clearance rate for clones carrying seven-fold mutations and as a consequence, accumulation of five- and six-fold mutations.

\section{Discussion}

The most frequent haplotype in the study area showed the following seven point mutations: $\operatorname{crt76T}$ crt326D crt356L crt220S dhfr59R dhfr108N mdr86Y. The high frequencies of these mutations were concordant with findings from previous studies in PNG [29,30]. Frequent mutations in $m d r 1$ and $c r t$ reflect the long history of 4-aminoquinoline usage in the country and the high levels of resistance against these drugs [42]. High frequencies of $d h f r$ mutations suggest that resistance to pyrimethamine is common and sulfadoxine, as indicated by the low level of mutations in $d h p s$, is probably the only effective component in the locally used first line treatment combination of CQ or Amodiaquine with SP.

The study presented here demonstrated different haplotype frequencies in subsets of the host population. Children five to 14 years of age harboured more seven-fold mutated haplotypes compared to adults. The same frequency of this haplotype was observed in new infections as in children. Assuming that new infections, as they were observed after radical cure, reflect the actually transmitted haplotypes via the Anopheline vector, these findings suggest three interpretations: (i) transmission of highly mutated and therefore probably more resistant haplotypes is modulated by drug pressure or other environmental factors so far undetected in this study; (ii) transmission of drug resistance markers occurs in an age-dependent mode by which mutant parasites accumulate in children;

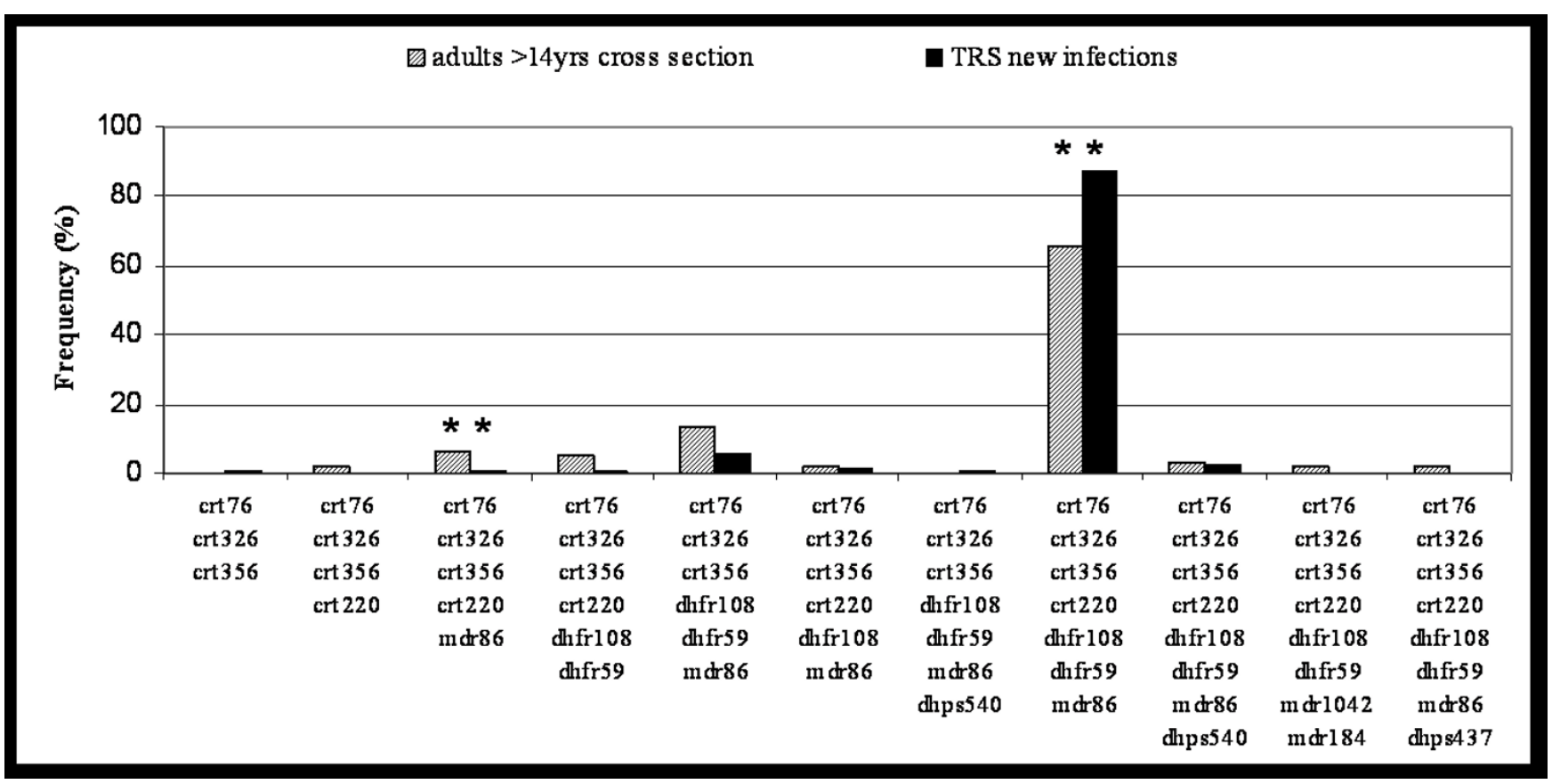

Figure 3

Comparison of haplotypes between adults (cross section) and new infections of the treatment to reinfection study. $*$ indicates significant difference of haplotype frequency between the two compared groups. 
or (iii), the parasite population found in children differs intrinsically from that in adults.

The first possible interpretation of the present findings implies that previous treatment effects the transmission of particular haplotypes. This is supported by the finding of higher prevalence and density of gametocytes following treatment with SP or CQ which was reported by a number of different authors [24-27], but is contradicted by a report from Dunyo et al [43] who could not find such an effect of SP treatment on subsequent transmission gametocyte carriage or density. In this study an altered transmission potential as a consequence to antimalarial treatment [44] was ruled out by excluding all individuals that had received antimalarial treatment two months prior to blood collection. Thus, effects of SP which is known to have a long elimination half life (4-9 days for Sulfadoxine and ca. 4 days for pyrimethamine) [45] should have waned. However, long lasting effects of SP treatment on transmission need to be further investigated.

An alternative explanation of the present findings implies that children and adults differ in their infectivity to mosquitoes. The age effect on transmission has been investigated by Graves and colleagues [46] in Madang, PNG, who performed direct mosquito feeding experiments on human blood. They found that the 1-20 year old individuals are more infectious to the mosquitoes than older age groups. However, a mathematical model developed by Ross et al [47] proposed that also infected adults are likely to make a substantial contribution to the infectious reservoir. The concordance of haplotype frequencies between new infections and children but not adults could suggest that children contribute more to transmission than adults in our study area. But such differential infectivity to mosquitoes is not the only explanation for the fact that frequencies in new infections do not differ from frequencies in age matched children. This could also be due to high turnover of infecting parasite clones in children. If in a particular age group clone acquisition rate is high, radically cured as well as non-cured individuals will all have predominantly recent infection and as a consequence will share the same haplotype frequencies of drug resistance markers.

As third possible explanation for heterogeneity in haplotype distribution other malariological parameters or host factors that have the potential to determine survival of the haplotypes in the host have to be considered. For example, $P$. falciparum infections in children differ from those in adults by a higher mean number of multiple infections $[40,48,49]$ and higher parasite densities $[49,50]$. A significantly reduced parasite density has been associated with resistance patterns [51]. In the data presented here, a reduced parasite density in mutant samples compared to wild type could not be confirmed. Also the densities of the two most common haplotypes did not differ between adults and children (data not shown). As further determinant of parasite survival in the host, some authors have proposed that mutations associated with drug resistance will incur fitness costs to the parasite in absence of drug pressure [51-55]. Since parasite fitness cannot be measured directly, a surrogate marker for fitness is required. The parameter "persistence of a clonal infection", measured as duration of an infection in a given host, would serve this purpose and can be measured experimentally in a longitudinal set of samples. This leads to the speculation that long-term persistence of a clonal infection in a host indicates better survival and thus could be used as a surrogate marker for parasite fitness.

Such fitness costs of drug resistance mutations can obviously only be studied in the absence of treatment. The effect of previous treatment on infections of particular haplotypes was ruled out in this dataset by excluding all individuals that had received antimalarial treatment 2 months prior to blood collection.

A recent paper of Ord and colleagues [56] reported that the prevalence of two mutations associated with chloroquine resistance declined during the dry season. The authors suggest fitness costs of drug resistance to be responsible for reduced survival of mutant parasites. A similar seasonal fluctuation has been suggested for Sudan [57]. These findings from a longitudinal study in a seasonal setting are perfectly in line with our findings from an area of perennial transmission where a higher frequency of mutated haplotypes was detected in new infections as compared to long lasting chronic infections.

In malaria endemic areas where transmission is perennial, most adults carry asymptomatic infections. These infections largely remain untreated and reflect chronic infections that persist over long periods of time (about 150 days) [40]. In case of long-term survival within a host, less mutated parasites would be expected to be more frequent due to their higher fitness. The present observation of a reduced frequency of the seven-fold mutant in adults compared to new infections is in support of reduced fitness of this particular haplotype. The opposite is true for less mutated haplotypes with increased prevalence in adults who frequently carry chronic subpatent parasitaemia.

When looking at each SNP separately, it was found that the frequency of the four most prevalent mutant and nonfixed SNPs (crt220S, dhfr59R, dhfr $108 \mathrm{~N}$ and $m d r 86 \mathrm{Y}$ ) was lower in chronic infections than in children or new infections. This also supports the hypothesis that infections harbouring fewer point mutations are fitter and can there- 
fore persist in the absence of drug pressure. The frequency of other SNPs was very low and does not allow for any interpretation.

The host's acquired immunity is an important parameter that needs to be considered when using the persistence of infection as a measure of parasite fitness. Immunity is a major determinant of duration of infection. However, the hosts acquired immunity is unlikely linked with a specific drug resistance haplotype as lack of linkage disequilibrium and sufficient outbreeding has been shown previously for the parasite population in the study area [33]. Therefore the immune response is expected to act on parasites irrespective of their number of drug resistance mutations.

Fitness costs of drug resistance is one important parameter for mathematical models that remains to be quantified in order to make more precise predictions on the spread of drug resistance. If point mutations incur fitness costs to the parasite in the absence of drug pressure, natural selection might lead to a decline in the prevalence of these mutations once the use of a specific drug is abolished. This might then result in the drug becoming efficacious again as has been observed in Malawi in the 12 years since CQ was removed from standard treatment [58]. Longitudinal studies are needed in order to estimate and quantify the reduction in survival of mutated versus wildtype genotypes in the host. This could provide more precise fitness measurements for parasites harbouring point mutations that are associated with drug resistance.

\section{Authors' contributions}

SS carried out the molecular genetic work and the statistical analysis. JM carried out the field survey and participated in the molecular genetic work. MG and MB carried out field work. IM was responsible for the treatment to reinfection study and participated in data analysis. IF was responsible for the study design and contributed to data analysis. All authors contributed to writing the manuscript.

\section{Acknowledgements}

We thank all the study participants, the IMR field team and microscopists. This study was supported by the Swiss National Research Foundation (SNF) (grant no:3 I00A0-I I I 196). S.S. was supported by the Forlen Foundation. The authors also thank AusAID for financial support.

\section{References}

I. Wu Y, Kirkman LA, Wellems TE: Transformation of Plasmodium falciparum malaria parasites by homologous integration of plasmids that confer resistance to pyrimethamine. Proc Natl Acad Sci U S A 1 996, 93: I I 30- I I 34.

2. Triglia T, Wang P, Sims PF, Hyde JE, Cowman AF: Allelic exchange at the endogenous genomic locus in Plasmodium falciparum proves the role of dihydropteroate synthase in sulfadoxineresistant malaria. EMBO J 1998, 17:3807-3815.
3. Sidhu $A B$, Verdier-Pinard D, Fidock DA: Chloroquine resistance in Plasmodium falciparum malaria parasites conferred by pfcrt mutations. Science 2002, 298:210-213.

4. Reed MB, Saliba KJ, Caruana SR, Kirk K, Cowman AF: Pgh I modulates sensitivity and resistance to multiple antimalarials in Plasmodium falciparum. Nature 2000, 403:906-909.

5. Babiker HA, Pringle SJ, bdel-Muhsin A, Mackinnon M, Hunt P, Walliker D: High-level chloroquine resistance in Sudanese isolates of Plasmodium falciparum is associated with mutations in the chloroquine resistance transporter gene pfort and the multidrug resistance Gene pfmdrl. J Infect Dis 200I, 183:1535-1538.

6. Djimde A, Doumbo OK, Cortese JF, Kayentao K, Doumbo S, Diourte Y, Dicko A, Su XZ, Nomura T, Fidock DA, Wellems TE, Plowe CV, Coulibaly D: A molecular marker for chloroquine-resistant falciparum malaria. N EnglJ Med 200I, 344:257-263.

7. Wellems TE, Plowe CV: Chloroquine-resistant malaria. J Infect Dis 200I, 184:770-776.

8. Basco LK, Le BJ, Rhoades Z, Wilson CM: Analysis of pfmdrl and drug susceptibility in fresh isolates of Plasmodium falciparum from subsaharan Africa. Mol Biochem Parasitol 1995, 74:157-166.

9. Nagesha HS, Din S, Casey GJ, Susanti AI, Fryauff DJ, Reeder JC, Cowman AF: Mutations in the pfmdrl, dhfr and dhps genes of Plasmodium falciparum are associated with in-vivo drug resistance in West Papua, Indonesia. Trans R Soc Trop Med Hyg 200I, 95:43-49.

10. L. S, Duraisingh MT, Drakeley C], Bailey R, Greenwood BM, Pinder M: Polymorphism of the Pfmdrl gene and chloroquine resistance in Plasmodium falciparum in The Gambia. Trans $R$ Soc Trop Med Hyg 1997, 9 I:450-453.

II. Basco LK, Tahar R, Keundjian A, Ringwald P: Sequence variations in the genes encoding dihydropteroate synthase and dihydrofolate reductase and clinical response to sulfadoxinepyrimethamine in patients with acute uncomplicated falciparum malaria. J Infect Dis 2000, 182:624-628.

12. Kublin JG, Dzinjalamala FK, Kamwendo DD, Malkin EM, Cortese JF, Martino LM, Mukadam RA, Rogerson SJ, Lescano AG, Molyneux ME, Winstanley PA, Chimpeni P, Taylor TE, Plowe CV: Molecular markers for failure of sulfadoxine-pyrimethamine and chlorproguanil-dapsone treatment of Plasmodium falciparum malaria. J Infect Dis 2002, 185:380-388.

13. Nzila AM, Mberu EK, Sulo J, Dayo H, Winstanley PA, Sibley CH, Watkins WM: Towards an understanding of the mechanism of pyrimethamine-sulfadoxine resistance in Plasmodium falciparum: genotyping of dihydrofolate reductase and dihydropteroate synthase of Kenyan parasites. Antimicrob Agents Chemother 2000, 44:991-996.

14. Talisuna AO, Nalunkuma-Kazibwe A, Langi P, Mutabingwa TK, Watkins WW, Van ME, Egwang TG, D'Alessandro U: Two mutations in dihydrofolate reductase combined with one in the dihydropteroate synthase gene predict sulphadoxine-pyrimethamine parasitological failure in Ugandan children with uncomplicated falciparum malaria. Infect Genet Evol 2004, 4:32I-327.

15. Hastings IM, Watkins WM: Intensity of malaria transmission and the evolution of drug resistance. Acta Trop 2005, 94:2। 8-229.

16. Hastings IM: Gametocytocidal activity in antimalarial drugs speeds the spread of drug resistance. Trop Med Int Health 2006, II:1206-I2I7.

17. Koella JC, Antia R: Epidemiological models for the spread of anti-malarial resistance. Malar J 2003, 2:3.

18. Laxminarayan R: Act now or later? Economics of malaria resistance. Am J Trop Med Hyg 2004, 7 1: 187-195.

19. Mackinnon MJ: Survival probability of drug resistant mutants in malaria parasites. Proc Biol Sci 1997, 264:53-59.

20. Mackinnon MJ, Hastings IM: The evolution of multiple drug resistance in malaria parasites. Trans $R$ Soc Trop Med Hyg 1998, 92: $188-195$

21. Dye C, Williams BG: Multigenic drug resistance among inbred malaria parasites. Proc Biol Sci 1997, 264:6I-67.

22. Gatton ML, Cheng Q: Plasmodium falciparum infection dynamics and transmission potential following treatment with sulfadoxine-pyrimethamine. J Antimicrob Chemother 2006, 58:47-5I.

23. Robert V, wono-Ambene HP, Le Hesran JY, Trape JF: Gametocytemia and infectivity to mosquitoes of patients with uncomplicated Plasmodium falciparum malaria attacks 
treated with chloroquine or sulfadoxine plus pyrimethamine. Am J Trop Med Hyg 2000, 62:210-216.

24. Schneider P, Bousema T, Omar S, Gouagna L, Sawa P, Schallig H, Sauerwein R: (Sub)microscopic Plasmodium falciparum gametocytaemia in Kenyan children after treatment with sulphadoxine-pyrimethamine monotherapy or in combination with artesunate. Int J Parasitol 2006, 36:403-408.

25. Sowunmi A, Fateye BA: Plasmodium falciparum gametocytaemia in Nigerian children: before, during and after treatment with antimalarial drugs. Trop Med Int Health 2003, 8:783-792.

26. Targett G, Drakeley C, Jawara M, von SL, Coleman R, Deen J, Pinder M, Doherty T, Sutherland C, Walraven G, Milligan P: Artesunate reduces but does not prevent posttreatment transmission of Plasmodium falciparum to Anopheles gambiae. J Infect Dis 200I, I 83: I254-I259.

27. L. S, Jawara M, Coleman R, Doherty T, Walraven G, Targett G: Parasitaemia and gametocytaemia after treatment with chloroquine, pyrimethamine/sulfadoxine, and pyrimethaminel sulfadoxine combined with artesunate in young Gambians with uncomplicated malaria. Trop Med Int Health 200I, 6:92-98.

28. Govere JM, Durrheim DN, Mngomezulu NM, Barnes K, Sharp B: Infectivity of Plasmodium falciparum gametocytes to Anopheles arabiensis after treatment with sulfadoxinepyrimethamine. Trans $R$ Soc Trop Med Hyg 2003, 97:707-708.

29. Casey GJ, Ginny M, Uranoli M, Mueller I, Reeder JC, Genton B, Cowman AF: Molecular analysis of Plasmodium falciparum from drug treatment failure patients in Papua New Guinea. Am J Trop Med Hyg 2004, 70:25 I-255.

30. Mita T, Kaneko A, Hwaihwanje I, Tsukahara T, Takahashi N, Osawa $\mathrm{H}$, Tanabe K, Kobayakawa T, Bjorkman A: Rapid selection of dhfr mutant allele in Plasmodium falciparum isolates after the introduction of sulfadoxine/pyrimethamine in combination with 4-aminoquinolines in Papua New Guinea. Infect Genet Evol 2006, 6:447-452.

31. Cortes A, Mellombo M, Benet A, Lorry K, Rare L, Reeder JC: Plasmodium falciparum: distribution of msp2 genotypes among symptomatic and asymptomatic individuals from the Wosera region of Papua New Guinea. Exp Parasitol 2004, 106:22-29.

32. Felger I, Tavul L, Kabintik S, Marshall V, Genton B, Alpers M, Beck HP: Plasmodium falciparum: extensive polymorphism in merozoite surface antigen 2 alleles in an area with endemic malaria in Papua New Guinea. Exp Parasitol 1994, 79: 106-1 I6.

33. Paul RE, Packer MJ, Walmsley M, Lagog M, Ranford-Cartwright LC, Paru R, Day KP: Mating patterns in malaria parasite populations of Papua New Guinea. Science 1995, 269:1709-17II.

34. Michon P, Cole-Tobian JL, Dabod E, Schoepflin S, Igu J, Susapu M, Tarongka N, Zimmerman PA, Reeder JC, Beeson JG, Schofield L, King $\mathrm{CL}$, Mueller I: The risk of malarial infections and disease in Papua New Guinean children. Am J Trop Med Hyg 2007, 76:997-1008.

35. McNamara DT, Kasehagen LJ, Grimberg BT, Cole-Tobian J, Collins WE, Zimmerman PA: Diagnosing infection levels of four human malaria parasite species by a polymerase chain reaction/ ligase detection reaction fluorescent microsphere-based assay. Am J Trop Med Hyg 2006, 74:4I 3-42I.

36. Felger I, Beck HP: Genotyping of Plasmodium falciparum. PCRRFLP analysis. Methods Mol Med 2002, 72:1 I7-129.

37. Irion A, Felger I, Abdulla S, Smith T, Mull R, Tanner M, Hatz C, Beck HP: Distinction of recrudescences from new infections by PCR-RFLP analysis in a comparative trial of CGP 56697 and chloroquine in Tanzanian children. Trop Med Int Health 1998, 3:490-497.

38. Snounou G: Genotyping of Plasmodium spp. Nested PCR. Methods Mol Med 2002, 72:103-116.

39. Crameri A, Marfurt J, Mugittu K, Maire N, Regos A, Coppee JY, Sismeiro O, Burki R, Huber E, Laubscher D, Puijalon O, Genton B, Felger I, Beck HP: Rapid microarray-based method for monitoring of all currently known single-nucleotide polymorphisms associated with parasite resistance to antimalaria drugs. I Clin Microbiol 2007, 45:3685-369|

40. Falk N, Maire N, Sama W, Owusu-Agyei S, Smith T, Beck HP, Felger I: Comparison of PCR-RFLP and Genescan-based genotyping for analyzing infection dynamics of Plasmodium falciparum. Am J Trop Med Hyg 2006, 74:944-950.
4I. Sama W, Dietz K, Smith T: Distribution of survival times of deliberate Plasmodium falciparum infections in tertiary syphilis patients. Trans R Soc Trop Med Hyg 2006, 100:8I I-8I6.

42. Muller I, Bockarie M, Alpers M, Smith T: The epidemiology of malaria in Papua New Guinea. Trends Parasitol 2003, 19:253-259.

43. Dunyo S, Milligan P, Edwards T, Sutherland C, Targett G, Pinder M: Gametocytaemia after drug treatment of asymptomatic Plasmodium falciparum. PLoS Clin Trials 2006, I:e20

44. Barnes KI, White $\mathrm{NJ}$ : Population biology and antimalarial resistance: The transmission of antimalarial drug resistance in Plasmodium falciparum. Acta Trop 2005, 94:230-240.

45. WHO: Guidelines for the treatment of malaria. 2006.

46. Graves PM, Burkot TR, Carter R, Cattani JA, Lagog M, Parker J, Brabin BJ, Gibson FD, Bradley DJ, Alpers MP: Measurement of malarial infectivity of human populations to mosquitoes in the Madang area, Papua, New Guinea. Parasitology 1988, 96 ( Pt 2):25I-263.

47. Ross A, Killeen G, Smith T: Relationships between host infectivity to mosquitoes and asexual parasite density in Plasmodium falciparum. Am J Trop Med Hyg 2006, 75:32-37.

48. Ntoumi F, Contamin H, Rogier C, Bonnefoy S, Trape JF, MercereauPuijalon O: Age-dependent carriage of multiple Plasmodium falciparum merozoite surface antigen-2 alleles in asymptomatic malaria infections. Am J Trop Med Hyg 1995, 52:8I-88.

49. Smith T, Beck HP, Kitua A, Mwankusye S, Felger I, Fraser-Hurt N, Irion A, Alonso P, Teuscher T, Tanner M: Age dependence of the multiplicity of Plasmodium falciparum infections and of other malariological indices in an area of high endemicity. Trans R Soc Trop Med Hyg 1999, 93 Suppl I:15-20.

50. Owusu-Agyei S, Smith T, Beck HP, menga-Etego L, Felger I: Molecular epidemiology of Plasmodium falciparum infections among asymptomatic inhabitants of a holoendemic malarious area in northern Ghana. Trop Med Int Health 2002, 7:421-428.

51. Osman ME, Mockenhaupt FP, Bienzle U, Elbashir MI, Giha HA: Fieldbased evidence for linkage of mutations associated with chloroquine (pfcrt/pfmdrl) and sulfadoxine-pyrimethamine (pfdhfr/pfdhps) resistance and for the fitness cost of multiple mutations in P. falciparum. Infect Genet Evol 2007, 7:52-59.

52. Hastings IM, Donnelly MJ: The impact of antimalarial drug resistance mutations on parasite fitness, and its implications for the evolution of resistance. Drug Resist Updat 2005, 8:43-50.

53. Kublin JG, Cortese JF, Njunju EM, Mukadam RA, Wirima JJ, Kazembe PN, Djimde AA, Kouriba B, Taylor TE, Plowe CV: Reemergence of chloroquine-sensitive Plasmodium falciparum malaria after cessation of chloroquine use in Malawi. J Infect Dis 2003, 187: 1870-1875

54. Walliker $\mathrm{D}$, Hunt $\mathrm{P}$, Babiker $\mathrm{H}$ : Fitness of drug-resistant malaria parasites. Acta Trop 2005, 94:25I-259.

55. Wang X, Mu J, Li G, Chen P, Guo X, Fu L, Chen L, Su X, Wellems TE: Decreased prevalence of the Plasmodium falciparum chloroquine resistance transporter $76 \mathrm{~T}$ marker associated with cessation of chloroquine use against $P$. falciparum malaria in Hainan, People's Republic of China. Am J Trop Med Hyg 2005, 72:410-414.

56. Ord R, Alexander N, Dunyo S, Hallett R, Jawara M, Targett G, Drakeley CJ, Sutherland CJ: Seasonal carriage of pfcrt and pfmdrI alleles in Gambian Plasmodium falciparum imply reduced fitness of chloroquine-resistant parasites. I Infect Dis 2007, 196:1613-1619.

57. Babiker HA, Satti G, Ferguson H, Bayoumi R, Walliker D: Drug resistant Plasmodium falciparum in an area of seasonal transmission. Acta Trop 2005, 94:260-268.

58. Laufer MK, Thesing PC, Eddington ND, Masonga R, Dzinjalamala FK, Takala SL, Taylor TE, Plowe CV: Return of chloroquine antimalarial efficacy in Malawi. N Engl J Med 2006, 355:1959-1966. 